\section{Infektionen durch Mycobacterium chimaera nach kardiochirurgischen Eingriffen}

van Ingen J et al. Global outbreak of severe Mycobacterium chimaera disease after cardiac surgery: a molecular epidemiological study. Lancet Infect Dis 2017; 17: 1033 - 1041

Seit dem Jahr 2013 wurden bei mehr als 100 Patienten nach kardiochirurgischen Eingriffen unter extrakorporaler Zirkulation schwere Endokarditiden von künstlichen Herzklappen durch Mycobacterium chimaera diagnostiziert, einige davon verliefen tödlich. Die Infektionen ereigneten sich in 4 europäischen Ländern (Schweiz, Deutschland, Niederlande, Großbritannien) sowie den USA und Australien.

Eingehende epidemiologische Untersuchungen identifizierten als wahrscheinliche Quelle der Erreger bestimmte Temperaturregulierungseinheiten (HeaterCooler Unit, HCU) der Herz-Lungen-Maschine. Diese HCUs bieten Mikroorganismen ausgezeichnete Wachstumsbedingungen, und da sie nicht luftdicht abgeschlossen sind, können die Ventilatoren der Einheiten erregerhaltige Aerosole in die Umgebung verbreiten. Um herauszufinden, wie die Erreger in die HCUs gelangt sind, hat eine europäische Arbeitsgruppe eine umfangreiche Detektivarbeit unternommen.

Dazu haben die Wissenschaftler um Jakko van Ingen Isolate von Mycobacterium chimaera verschiedener Herkunft gewonnen:

- von den europäischen Patienten mit Infektion nach kardiochirurgischen Eingriffen (24 Isolate von 21 Patienten),

- von unterschiedlichen HCU-Typen in Krankenhäusern und von den Produktionsstätten von 2 Herstellern (181 Isolate),

- Isolate von Patienten mit pulmonalen Mycobacterium-chimaera-Infektionen, ohne Beziehung zur Kardiochirurgie,
- Isolate aus der Umwelt in den teilnehmenden Krankenhäusern, z. B. aus Leitungswasser, Trinkwasserspendern und der Luft, ohne Beziehung zur Kardiochirurgie, und

- Isolate von Mycobacterium intracellulare, das genetisch Mycobacterium chimaera am nächsten steht.

Insgesamt 250 Proben wurden dann mittels Whole Genome Sequencing analysiert und die Ergebnisse untereinander und mit denen veröffentlichter Genome von Mycobacterium chimaera verglichen.

Dabei fanden die Forscher 2 Hauptgruppen von Mycobacterium chimaera: Bis auf 1 Ausnahme gehörten alle Isolate der kardiochirurgischen Patienten zur Gruppe 1 und zeigten nur eine geringe genetische Diversität. In diese Untergruppe ließen sich auch die Ergebnisse von 11 Patienten aus den USA einordnen, die bereits veröffentlicht waren. Und schließlich befanden sich in dieser Gruppe die meisten Isolate von HCUs eines der beiden Hersteller sowie 1 Isolat von einer Produktionsstätte des gleichen Herstellers. Die Isolate der HCUs des anderen Herstellers ebenso wie die der Patienten mit pulmonalen Infektionen unterschieden sich dagegen deutlich stärker voneinander.

Der oft schleichende Verlauf der Endokarditiden lässt es möglich erscheinen, dass zukünftig noch weitere Infektionen diagnostiziert werden könnten - Stichtag für die hier vorgestellten Daten war der 1. Juli 2015. Kliniker sollten bei Patienten mit anamnestisch bekannten kardiochirurgischen Eingriffen und eingeschränktem Immunstatus diese Möglichkeit in Betracht ziehen.
FAZIT

Die Ergebnisse dieser umfassenden epidemiologischen molekulargenetischen Untersuchung lassen vermuten, dass die große Mehrzahl der Mycobacterium-chimaera-Infektionen auf eine Verunreinigung schon bei der Herstellung der verwendeten HCUs an einem Produktionsort zurückgeht, so die Autoren. Allerdings fanden sich auch lokale HCU-Kontaminationen, und 1 Patient hat sich vermutlich auf diesem Weg infiziert.

Dr. Elke Ruchalla, Bad Dürrheim 